\title{
ON THE EFFICIENCY OF RANDOM WALK APPROACH TO NOISE REDUCTION IN COLOR IMAGES
}

\author{
B. Smolka* M. Szczepanski* \\ Silesian University of Technology \\ Department of Automatic Control \\ Akademicka 16 Str, 44-101 Gliwice, Poland \\ bsmolka@ia.polsl.gliwice.pl
}

\author{
K.N. Plataniotis A. N. Venetsanopoulos \\ Edward S. Rogers Sr. Department of \\ Electrical and Computer Engineering \\ University of Toronto \\ 10 King's College Road, Toronto, Canada \\ kostasadsp.toronto. edu
}

\begin{abstract}
In this paper we propose a new algorithm of noise reduction in color images. The new technique of multichannel image enhancement is capable of reducing impulse and Gaussian noise and it outperforms the basic methods based on vector median used for the noise reduction in color images. In the paper a new smoothing operator, based on a random walk model and on a fuzzy similarity measure between pixels connected by a digital geodesic path is introduced. The efficiency of the proposed method was tested on the standard color images using the widely used objective image quality measures.
\end{abstract}

\section{STANDARD NOISE REDUCTION FILTERS}

Most popular nonlinear, multichannel filters are based on the ordering of vectors in a predefined moving window [16]. The output of these filters is defined as the lowest ranked vector according to a specific vector ordering technique.

Let $\mathbf{F}(x)$ : represent a multichannel image and let $W$ be a window of finite size $n$ (filter length). The noisy image vectors inside the filtering window $W$ are denoted as $\mathbf{F}_{j}, j=0,1, \ldots, n-1$. If the distance between two vectors $\mathbf{F}_{i}, \mathbf{F}_{j}$ is denoted as $\rho\left(\mathbf{F}_{i}, \mathbf{F}_{j}\right)$ then the scalar quantity $R_{i}=\sum_{j=0}^{n-1} \rho\left(\mathbf{F}_{i}, \mathbf{F}_{j}\right)$, is the distance associated with the noisy vector $\mathbf{F}_{i}$. The ordering of the $R_{i}$ 's: $R_{(1)} \leq$ $\ldots \leq R_{(n-1)}$, implies the same ordering to the corresponding vectors $\mathbf{F}_{i}: \mathbf{F}_{(1)} \leq \ldots \leq \mathbf{F}_{(n-1)}$. Nonlinear ranked type multichannel estimators define the vector $\mathbf{F}_{(0)}$ as the filter output. However, the concept of input ordering, initially applied to scalar quantities is not easily extended to multichannel data, since there is no universal way to define ordering in vector spaces.

To overcome this problem, distance functions are often utilized to order vectors. As an example, the Vector Median Filter (VMF) uses the $L_{1}$ or $L_{2}$ norm to order vectors according to their relative magnitude differences. The orien-

\footnotetext{
* This work was partially supported by KBN grant 8 T11E 01319
}

tation difference between two vectors can also be used to remove vectors with atypical directions (Vector Directional Filter - VDF, Basic Vector Directional Filters- BVDF).

The reduction of image noise without major degradation of the image structure is one of the most important problems of the low-level image processing. A whole variety of algorithms has been developed, but none of them can be seen as a final solution of the noise problem and therefore a new filtering technique, which copes better with impulsive and Gaussian noise has been proposed.

\section{NEW ALGORITHM OF NOISE REDUCTION}

Let us assume, that $R^{2}$ is the Euclidean space, $W$ is a planar subset of $R^{2}$ and $x, y$ are points of of the set $W$.

A path from $x$ to $y$ is a continuous mapping $\mathcal{P}:[a, b] \rightarrow$ $X$, such that $\mathcal{P}(a)=x$ and $\mathcal{P}(b)=y$. Point $x$ is the starting point and $y$ is the end point of the path $\mathcal{P}$ [8-10].

An increasing polygonal line $P$ on the path $\mathcal{P}$ is any polygonal line $P=\left\{g\left(\lambda_{i}\right)\right\}_{i=0}^{n}, a=\lambda_{0}<\ldots<\lambda_{n}=b$. The length of the polygonal line $P$ is the total sum of its constitutive line segments $L(P)=\sum_{i=1}^{n} \rho\left(\mathcal{P}\left(\lambda_{i-1}, \lambda_{i}\right)\right.$, where $\rho(x, y)$ is the distance between the points $x$ and $y$, when a specific metric is adopted.

If $\mathcal{P}$ is a path from $x$ to $y$ then it is called rectifiable, if and only if $L(P)$, where $P$ is an increasing polygonal line is bounded. Its upper bound is called the length of the path $\mathcal{P}$. The geodesic distance $\rho^{W}(x, y)$ between points $x$ and $y$ is the lower bound of the length of all paths leading from $x$ to $y$ totally included in $W$. If such paths do not exist, then the value of the geodesic distance is set to $\infty$. The geodesic distance verifies $\rho^{W}(x, y) \geq \rho(x, y)$ and in the case when $W$ is a convex set then $\rho^{W}(x, y)=\rho(x, y)$.

The notion of the geodesic distance can be extended to a lattice, which is a set of discrete points, in our case image pixels.

Let a digital lattice $\mathcal{H}=(\mathbf{F}, \mathcal{N})$ be defined by $\mathbf{F}$, which is the set of all points of the plane (pixels of a color im- 
age) and the neighbourhood relation $\mathcal{N}$ between the lattice points.

A digital path $P=\left\{p_{i}\right\}_{i=0}^{n}$ on the lattice $\mathcal{H}$ is a sequence of neighbouring points $\left(p_{i-1}, p_{i}\right) \in \mathcal{N}$. The length $L(P)$ of digital path $P\left\{p_{i}\right\}_{i=0}^{n}$ is simply $\sum_{i=1}^{n} \rho^{\mathcal{H}}\left(p_{i-1}, p_{i}\right)$. If $P(x, y)$ denotes the digital path connecting the points $x$ and $y$ in $F$ then the lattice distance between those points is defined as $\rho^{\mathcal{H}}(x, y)=\min _{P(x, y)} L[P(x, y)]$.

Constraining the paths to be totally included in a predefined set $W \in \mathbf{F}$ yields the digital geodesic distance $\rho^{W}$. In this paper we will assign to the distance of neighbouring points the value 1 and will be working with the 8 neighbourhood system.

Let the pixels $(i, j)$ and $(k, l)$ be called connected, denoted as $(i, j) \leftrightarrow(k, l)$, if there exists a geodesic path $P^{W}\{(i, j),(k, l)\}$ contained in the set $W$ starting from $(i, j)$ and ending at $(k, l)$ (Fig. 1).

If two pixels $\left(x_{0}, y_{0}\right)$ and $\left(x_{n}, y_{n_{n}}\right)$ are connected by a geodesic path $P^{W}\left\{\left(x_{0}, y_{0}\right),\left(x_{1}, y_{1}\right), \ldots,\left(x_{n}, y_{n}\right)\right\}$ of length $n$ then let $x$

$\chi^{W, n}\left\{\left(x_{0}, y_{0}\right),\left(x_{n}, y_{n}\right)\right\}=\sum_{k=0}^{n_{t}-1}\left\|\mathbf{F}\left(x_{k+1}, y_{k+1}\right)-\mathbf{F}\left(x_{k}, y_{k}\right)\right\|$

be a measure of dissimilarity between pixels $\left(x_{0}, y_{0}\right)$ and $\left(x_{n}, y_{n}\right)$, along a specific geodesic path $P^{W}$ joining $\left(x_{0}, y_{0}\right)$ and $\left(x_{n}, y_{n}\right)$. If at path joining two distinct points $x, y$, such that $\mathbf{F}(x)=\mathbf{F}(y)$ consists of lattice points of the same values, then $\chi^{W, n}(x, y)=0$ otherwise $\chi^{W, n}(x, y)>0$.

Let us now define the similarity function between two pixels connected along all geodesic digital paths leading from $(i, j)$ and $(k, l)$ (Fig. 1) [7]

$\mu^{W, n}\{(i, j),(k, l)\}=\frac{1}{\omega} \sum_{l=1}^{\omega} \exp \left[-\beta \cdot \chi_{l}^{W, n}\{(i, j),(k, l)\}\right]$

where $\omega$ is the number of all geodesic paths connecting $(i, j)$ and $(k, l), \beta$ is a parameter and $\chi_{l}^{W n}\{(i, j),(k, l)\}$ is a dissimilarity value along a specific path from a set of all $\omega$ possible paths leading from $(i, j)$ to $(k, l)$. In this way $\mu^{W, \pi}\{(i, j),(k, l)\}$ is an average value, taken over all routes joining the starting point $(i, j)$ and the end point $(k, l)$.

\begin{tabular}{|c|c|c|c|c|c|c|c|c|c||c|c|c|}
\hline$q$ & $-q$ & $q$ & $q$ & $q$ & $q$ & $q$ & $q$ & $q$ & $q$ & $q$ & $q$ \\
\hline$q$ & $q$ & $q$ & $q$ & $q$ & $q$ & $q$ & $q$ & $q$ & $q$ & $q$ & $q$ & $q$ \\
\hline$q$ & $q$ & $q$ & $q$ & $q$ & $q$ & $q$ & $q$ & $q$ & $q$ & $q$ & $q$ \\
\hline
\end{tabular}

Fig. 1. There are four geodesic paths of length 2 connecting two neighbouring points contained in the $3 \times 3$ window $W$ when the 8-neighbourhood system is applied.
For $n=1$ and $W$ a square mask of the size $3 \times 3$, we have $\left.\mu^{W, 1}\{(i, j),(k, l)\}=\exp \{-\beta\|\mathbf{F}(i, j)-\mathbf{F}(k, l)\|\}\right\}$ and when $\mathbf{F}(i, j)=\mathbf{F}(k, l)$ then $\chi^{W, n}\{(i, j),(k, l)\}=0$, $\mu\{(i, j),(k, l)\}=1$, and for $\|\mathbf{F}(i, j)-\mathbf{F}(k, l)\| \rightarrow \infty$ then $\mu \rightarrow 0$.

The normalized similarity function takes the form

$$
\psi^{W, n}\{(i, j),(k, l)\}=\frac{\mu^{W, n}\{(i, j),(k, l)\}}{\sum_{(l, m) \Leftrightarrow(i, j)} \mu^{W, n}\{(i, j),(l, m)\}}
$$

The normalized similarity function has the property

$$
\sum_{(k, l) \Leftrightarrow(i, j)} \psi^{W, n}\{(i, j),(k, l)\}=1
$$

Now we are in a position to define a smoothing transformation $\mathcal{J}$

$$
\mathbf{J}(i, j)=\sum_{(k, l) \Leftrightarrow(i, j)} \psi^{W, n}\{(i, j),(k, l)\} \cdot \mathbf{F}(k, l)
$$

where $(k, l)$ are points which are connected with $(i, j)$ by geodesic digital paths of length $n$ included in $W$.

\section{RESULTS}

The effectiveness of the new smoothing operator defined by (5) was tested using the LENA and PEPPERS standard images contaminated by Gaussian noise of $\sigma=30$. We also used the LENA image contaminated by $4 \%$ impulsiv noise (salt \& pepper added on each channel) mixed with Gaussian noise $(\sigma=30)$.

The performance of the presented method was evaluated by means of the objective image quality measures RMSE, PSNR, NMSE and NCD [3]. Tables 2 and 3 show the obtained results for $n=3$ and $\beta$ increasing linearly from 10 to 30 . After 3 iterations the filtered image was being sharpened and was visually more pleasing, however the quality measures were decreasing. Therefore only the results of 3 iterations are shown in the Tab. 2 and 3. Additionally Fig. 2 shows the comparison of the new filtering technique with the standard vector median.

For the calculation of the similarity function we used the $L_{1}$ metric and an exponential function, however we have obtained good results using other convex functions and different vector metrics.

\section{CONCLUSIONS}

In this paper, a new filter for noise reduction in color images has been presented. Experimental results indicate that the new filtering technique outperforms standard procedures used to reduce mixed impulsive and Gaussian noise in color images. The efficiency of the new filtering technique is shown in Tables 2 and 3. 


\begin{tabular}{|c|c|}
\hline Notation & Filter \\
\hline AMF & Arithmetic Mean Filter \\
\hline VMF & Vector Median Filter \\
\hline BVDF & Basic Vector Directional Filter \\
\hline GVDF & Generalized Vector Directional Filter \\
\hline DDF & Directional-Distance Filter \\
\hline HDF & Hybrid Directional Filter \\
\hline AHDF & Adaptive Hybrid Directional Filter \\
\hline FVDF & Fuzzy Vector Directional Filter \\
\hline ANNF & Adaptive Nearest Neighbor Filter \\
\hline ANP-EF & Adaptive Non Parametric (Exponential) Filter \\
\hline ANP-GF & Adaptive Non Parametric (Gaussian) Filter \\
\hline ANP-DF & Adaptive Non Parametric (Directional) Filter \\
\hline VBAMMF & Vector Bayesian Adaptive Median/Mean Filter \\
\hline
\end{tabular}

Table 1. Filters taken for comparison with the proposed filter [1-5].

\section{REFERENCES}

[1] A.N. Venetsanopoulos, K.N. Plataniotis, Multichannel image processing, Proceedings of the IEEE Workshop on Nonlinear Signal/Image Processing, 26, (1995)

[2] I. Pitas, A. N. Venetsanopoulos, 'Nonlinear Digital Filters : Principles and Applications', Kluwer Academic Publishers, Boston, MA, (1990)

[3] K.N. Plataniotis, A.N. Venetsanopoulos, 'Color Image Processing and Applications', Springer Verlag, (June 2000)

[4] I. Pitas, P. Tsakalides, Multivariate ordering in color image processing, IEEE Trans. on Circuits and Systems for Video Technology, 1, 3, 247-256, (1991)

[5] I. Pitas, A.N. Venetsanopoulos, Order statistics in digital image processing, Proceedings of IEEE, 80, 12, 1893-1923, (1992)

[6] J. Astola, P. Haavisto, Y. Neuovo, Vector median filters, IEEE Proceedings, 78, 678-689, (1990)

[7] 12. B. Smolka, K. Wojciechowski, Random walk approach to image enhancement, Signal Processing, Vol. 81 , No. 4

[8] G. Borgefors, Distances transformations in digital images. Computer Vision, Graphics and Image Processing, 34:334-371,1986

[9] G. Matheron, Random Sets and Integral Geometry. John Willey, New York, 1975

[10] Henk J.A.M. Heijmans., Mathematical Morphology: Basic Principles, Proceedings of the Summer School on Morphological Image and Signal Processing, Zakopane, Poland, 1995
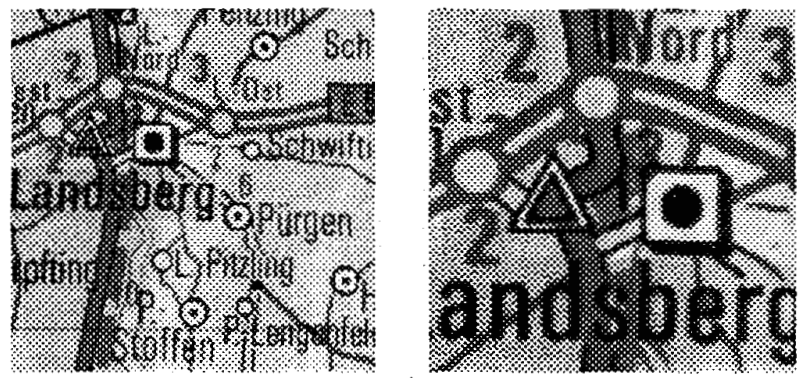

a)
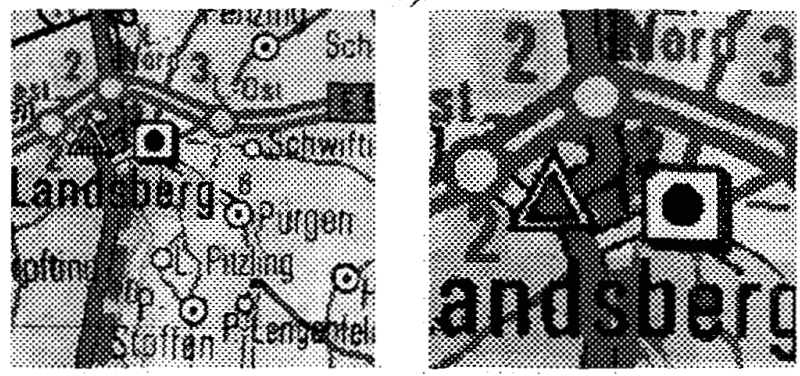

b)
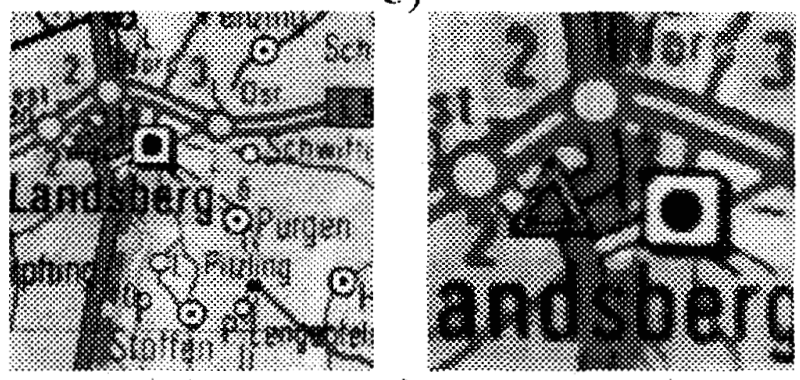

c)
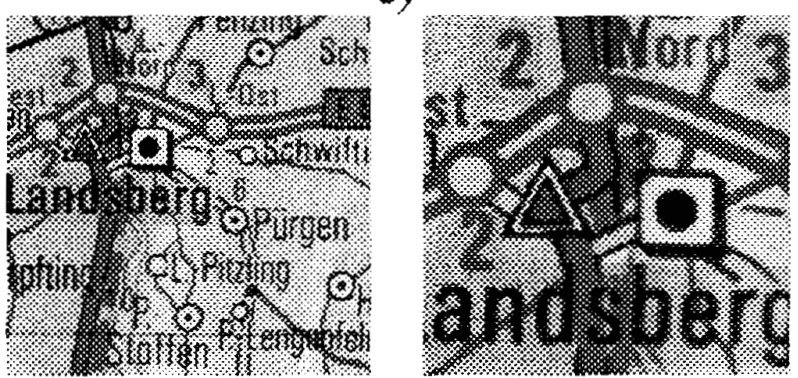

d)

Fig. 2. Comparison of the efficiency of the vector median and the proposed filter: a) test image (part of a scanned map), b) result of the new filtering technique ( $\beta=20, \alpha=$ $1.25,3$ iterations $), c)$ result of the standard vector median filtration $(3 \times 3$ mask $)$, c) result of the DDF $(3 \times 3$ mask $)$ 


\begin{tabular}{|c|c|c|c|c|c|}
\hline METHOD $_{N}$ & $\begin{array}{l}\text { NMSE } \\
{\left[10^{-3}\right]}\end{array}$ & RMSE & $\begin{array}{l}\text { SNR } \\
\text { [dB] }\end{array}$ & $\begin{array}{c}\text { PSNR } \\
\text { [dB] }\end{array}$ & $\begin{array}{c}\text { NCD } \\
{\left[10^{-4}\right]}\end{array}$ \\
\hline NONE & 502.410 & 28.683 & 12.989 & 18.978 & 244.190 \\
\hline $\mathrm{AMF}_{1}$ & 90.184 & 12.152 & 20.449 & 26.438 & 115.210 \\
\hline $\mathrm{AMF}_{3}$ & 88.815 & 12.060 & 20.515 & 26.504 & 99.043 \\
\hline $\mathrm{AMF}_{5}$ & 113.840 & 13.653 & 19.437 & 25.426 & 98.853 \\
\hline$\overline{\text { VIF }_{1}}$ & 168.830 & 16.627 & 17.725 & 23.714 & 158.920 \\
\hline $\mathrm{VMF}_{3}$ & 113.420 & 13.628 & 19.453 & 25.442 & 129.700 \\
\hline $\mathrm{VMF}_{5}$ & 105.180 & 13.124 & 19.781 & 25.770 & 123.390 \\
\hline $\mathrm{BVDF}_{1}$ & 372.320 & 24.691 & 14.291 & 20.280 & 153.420 \\
\hline $\mathrm{BVDF}_{3}$ & 363.390 & 24.394 & 14.396 & 20.385 & 129.040 \\
\hline BVDF $_{5}$ & 367.740 & 24.539 & 14.345 & 20.334 & 124.350 \\
\hline GVDF $_{1}$ & 144.640 & 15.390 & 18.397 & 24.386 & 127.370 \\
\hline GVDF $_{3}$ & 99.400 & 12.758 & 20.026 & 26.015 & 97.348 \\
\hline GVDF $_{5}$ & 100.490 & 12.828 & 19.979 & 25.968 & 92.583 \\
\hline$\overline{\overline{D D F}_{1}}$ & 184.620 & 17.387 & $\begin{array}{l}17.337 \\
\end{array}$ & 23.326 & 149.540 \\
\hline$\overline{\mathrm{DDF}}_{3}$ & 127.260 & 14.436 & 18.953 & 24.942 & 120.400 \\
\hline $\mathrm{DDF}_{5}$ & 118.820 & 13.949 & 19.251 & 25.240 & 114.400 \\
\hline $\mathrm{HDF}_{1}$ & 147.060 & 15.518 & 18.325 & 24.314 & 139.380 \\
\hline $\mathrm{HDF}_{3}$ & 87.730 & 11.986 & 20.569 & 26.558 & 107.600 \\
\hline $\mathrm{HDF}_{5}$ & 79.698 & 11.424 & 20.986 & 26.975 & 101.140 \\
\hline $\mathrm{AHDF}_{1}$ & 131.390 & 14.668 & 18.814 & 24.803 & 137.650 \\
\hline AHDF $_{3}$ & 78.739 & 11.355 & 21.038 & 27.027 & 106.180 \\
\hline $\mathrm{AHDF}_{5}$ & 72.331 & 10.883 & 21.407 & 27.396 & 99.673 \\
\hline$\overline{\text { FVDF }}_{1}$ & 103.950 & 13.047 & 19.832 & 25.821 & 112.450 \\
\hline $\mathrm{FVDF}_{3}$ & 72.888 & 10.925 & 21.373 & 27.362 & 89.743 \\
\hline $\mathrm{FVDF}_{5}$ & 77.012 & 11.230 & 21.134 & 27.123 & 88.023 \\
\hline $\mathrm{ANNF}_{1}$ & 112.660 & 13.583 & 19.482 & 25.471 & 120.270 \\
\hline $\mathrm{ANNF}_{3}$ & 80.934 & 11.512 & 20.919 & 26.908 & 96.789 \\
\hline $\mathrm{ANNF}_{5}$ & 84.101 & 11.735 & 20.752 & 26.741 & 93.171 \\
\hline ANP-E 1 & 88.827 & 12.060 & 20.515 & 26.504 & 115.100 \\
\hline $\mathrm{ANP}_{3} \mathrm{E}_{3}$ & 79.688 & 11.423 & 20.986 & 26.975 & 100.860 \\
\hline ANP-E & 94.793 & 12.459 & 20.232 & 26.221 & 101.070 \\
\hline ANP-G 1 & 88.787 & 12.058 & 20.517 & 26.506 & 115.080 \\
\hline ANP-G 3 & 79.674 & 11.422 & 20.987 & 26.976 & 100.850 \\
\hline ANP-G 5 & 94.741 & 12.455 & 20.235 & 26.224 & 101.050 \\
\hline ANP-D 1 & 105.280 & 13.130 & 19.776 & 25.765 & 113.610 \\
\hline ANP-D 3 & 73.211 & 10.949 & 21.354 & 27.343 & 89.078 \\
\hline ANP-D 5 & 78.419 & 11.332 & 21.056 & 27.045 & 87.650 \\
\hline$\overline{\text { VBAMMF }}_{1}$ & 90.184 & 12.152 & 20.449 & 26.438 & 115.210 \\
\hline VBAMMF $_{3}$ & 88.815 & 12.060 & 20.515 & 26.504 & 99.043 \\
\hline VBAMMF $_{5}$ & 113.840 & 13.653 & 19.437 & 25.426 & 98.853 \\
\hline NEW $_{1}$ & 65.412 & 10.349 & 21.843 & 27.832 & 95.248 \\
\hline $\mathrm{NEW}_{2}$ & 57.921 & 9.739 & 22.372 & 28.361 & 88.917 \\
\hline $\mathrm{NEW}_{3}$ & 61.473 & 10.033 & 22.113 & 28.102 & 88.561 \\
\hline
\end{tabular}

Table 2. Comparison of the new algorithm with the standard techniques (Tab. 1) using the PEPPERS standard image corrupted by Gaussian noise $\sigma=30$. The subscripts denote the iteration number.

\begin{tabular}{|c|c|c|c|c|c|}
\hline $\operatorname{METHOD}_{N}$ & $\begin{array}{l}\text { NMSE } \\
{\left[10^{-3}\right]}\end{array}$ & RMSE & $\begin{array}{l}\text { SNR } \\
\text { [dB] }\end{array}$ & $\begin{array}{c}\text { PSNR } \\
{[\mathrm{dB}]}\end{array}$ & $\begin{array}{c}\mathrm{NCD} \\
{\left[10^{-4}\right]}\end{array}$ \\
\hline NONE & 905.930 & 42.674 & 10.429 & 15.528 & 305.550 \\
\hline $\mathrm{AMF}_{1}$ & 128.940 & 16.099 & $\overline{18.896}$ & 23.995 & 122.880 \\
\hline $\mathrm{AMF}_{3}$ & 97.444 & 13.996 & 20.112 & 25.211 & 95.800 \\
\hline $\mathrm{AMF}_{5}$ & 113.760 & 15.122 & 19.440 & 24.539 & 92.312 \\
\hline $\mathrm{VMF}_{1}$ & 161.420 & 18.013 & 17.920 & 23.019 & 161.700 \\
\hline $\mathrm{VMF}_{3}$ & 104.280 & 14.478 & 19.818 & 24.916 & 128.620 \\
\hline $\mathrm{VMF}_{5}$ & 96.464 & 13.925 & 20.156 & 25.255 & 121.790 \\
\hline$\overline{\text { BVDF }_{1}}$ & 354.450 & 26.692 & 14.504 & 19.603 & $\overline{152.490}$ \\
\hline $\mathrm{BVDF}_{3}$ & 336.460 & 26.006 & 14.731 & 19.829 & 123.930 \\
\hline $\mathrm{BVDF}_{5}$ & 338.940 & 26.102 & 14.699 & 19.797 & 118.500 \\
\hline GVDF $_{1}$ & 140.970 & 16.833 & 18.509 & 23.607 & 126.820 \\
\hline $\mathrm{GVDF}_{3}$ & 93.444 & 13.705 & 20.294 & 25.393 & 94.627 \\
\hline $\mathrm{GVDF}_{5}$ & 91.118 & 13.534 & 20.404 & 25.503 & 89.277 \\
\hline$\overline{\text { DDF }_{1}}$ & 176.670 & 18.845 & 17.528 & 22.627 & 152.050 \\
\hline$\overline{\mathrm{DDF}_{3}}$ & 119.330 & 15.488 & 19.232 & 24.331 & 119.940 \\
\hline$\overline{\mathrm{DDF}}_{5}$ & 110.620 & 14.912 & 19.561 & 24.660 & 113.390 \\
\hline$\overline{\mathrm{HDF}_{1}}$ & 143.190 & 16.966 & $\overline{18.441}$ & 23.539 & 139.360 \\
\hline $\mathrm{HDF}_{3}$ & 82.413 & 12.871 & 20.840 & 25.939 & 104.620 \\
\hline $\mathrm{HDF}_{5}$ & 74.487 & 12.236 & 21.279 & 26.378 & 97.596 \\
\hline$\overline{\text { AHDF }_{1}}$ & 132.710 & 16.333 & 18.771 & 23.869 & 138.180 \\
\hline $\mathrm{AHDF}_{3}$ & 75.236 & 12.298 & 21.236 & 26.334 & 103.410 \\
\hline $\mathrm{AHDF}_{5}$ & 68.563 & 11.740 & 21.639 & 26.738 & 96.327 \\
\hline$\overline{\text { FVDF }_{1}}$ & 108.760 & 14.786 & 19.635 & 24.734 & 111.220 \\
\hline $\mathrm{FVDF}_{3}$ & 73.796 & 12.179 & 21.320 & 26.418 & 83.629 \\
\hline FVDF $_{5}$ & 76.274 & 12.382 & 21.176 & 26.275 & 80.081 \\
\hline ANNF $_{1}$ & 110.720 & 14.919 & 19.558 & 24.656 & 113.560 \\
\hline $\mathrm{ANNF}_{3}$ & 75.652 & 12.332 & 21.212 & 26.310 & 86.836 \\
\hline $\mathrm{ANNF}_{5}$ & 76.757 & 12.421 & 21.149 & 26.247 & 82.825 \\
\hline ANP-E 1. & 128.590 & 16.077 & 18.908 & 24.007 & 122.890 \\
\hline ANP-E 3 & 90.509 & 13.488 & 20.433 & 25.532 & 97.621 \\
\hline ANP-E $_{5}$ & 96.930 & 13.959 & 20.135 & 25.234 & 94.131 \\
\hline$\overline{A N P}-G_{1}$ & 128.600 & 16.078 & 18.908 & 24.006 & 122.900 \\
\hline ANP-G & 90.523 & 13.489 & 20.432 & 25.531 & 97.603 \\
\hline ANP-G 5 & 96.990 & 13.963 & 20.133 & 25.231 & 94.134 \\
\hline ANP-D 1 & 113.900 & 15.131 & 19.435 & 24.533 & 115.230 \\
\hline ANP-D 3 & 74.203 & 12.213 & 21.296 & 26.394 & 85.026 \\
\hline $\mathrm{ANP}-\mathrm{D}_{5}$ & 76.265 & 12.381 & 21.177 & 26.275 & 81.202 \\
\hline VBAMMF $_{1}$ & 128.940 & 16.099 & 18.896 & 23.995 & 122.880 \\
\hline VBAMMF $_{3}$ & 97.444 & 13.996 & 20.112 & 25.211 & 95.800 \\
\hline VBAMMF $_{5}$ & 113.760 & 15.122 & 19.440 & 24.539 & 92.312 \\
\hline$\overline{\text { NEW }_{1}}$ & 74762 & 12.259 & 21.263 & 26.362 & $8 \overline{83.585}$ \\
\hline $\mathrm{NEW}_{2}$ & 55.239 & 10.537 & 22578 & 27.676 & 72.115 \\
\hline $\mathrm{NEW}_{3}$ & 56.078 & 10.617 & 22.512 & 27.611 & 70.008 \\
\hline
\end{tabular}

Table 3. Comparison of the new algorithm with the standard techniques (Tab. 1) using the LENA standard image corrupted by $4 \%$ Impulse and Gaussian noise $\sigma=30$. The subscripts denote the iteration number. 